Check for updates

Cite this: J. Mater. Chem. A, 2019, 7, 843

DOI: $10.1039 / c 8 t a 90285 b$

www.rsc.org/MaterialsA

\section{Correction: Engineering tin phosphides@carbon yolk-shell nanocube structures as a highly stable anode material for sodium-ion batteries}

\author{
Lianbo Ma, ${ }^{\text {ab }}$ Pengjie Yan, ${ }^{\mathrm{b}}$ Shikui Wu, ${ }^{\text {bc }}$ Guoyin Zhu*a and Yalong Shen*bd
}

Correction for 'Engineering tin phosphides@carbon yolk-shell nanocube structures as a highly stable anode material for sodium-ion batteries' by Lianbo Ma et al., J. Mater. Chem. A, 2017, 5, 16994-17000.

In the description "As presented in Fig. $\mathrm{S} 3$, both $\mathrm{Sn}_{4} \mathrm{P}_{3} / \mathrm{C}$ and bare $\mathrm{Sn}_{4} \mathrm{P}_{3}$ materials exhibit much bigger particle sizes, which are ascribed to the aggregation of Sn below its melting point" on p16997 of the published article, the reference to 'Fig. S3' should instead read 'Fig. S2'.

Moreover, in the published version of Fig. S2, the TEM images are wrong and may have been mixed with those of other samples. To avoid any possible misleading, these samples have been prepared and characterized again. The authors would like to update the Fig. S2 with a corrected version, containing new TEM and XRD data, as shown below.
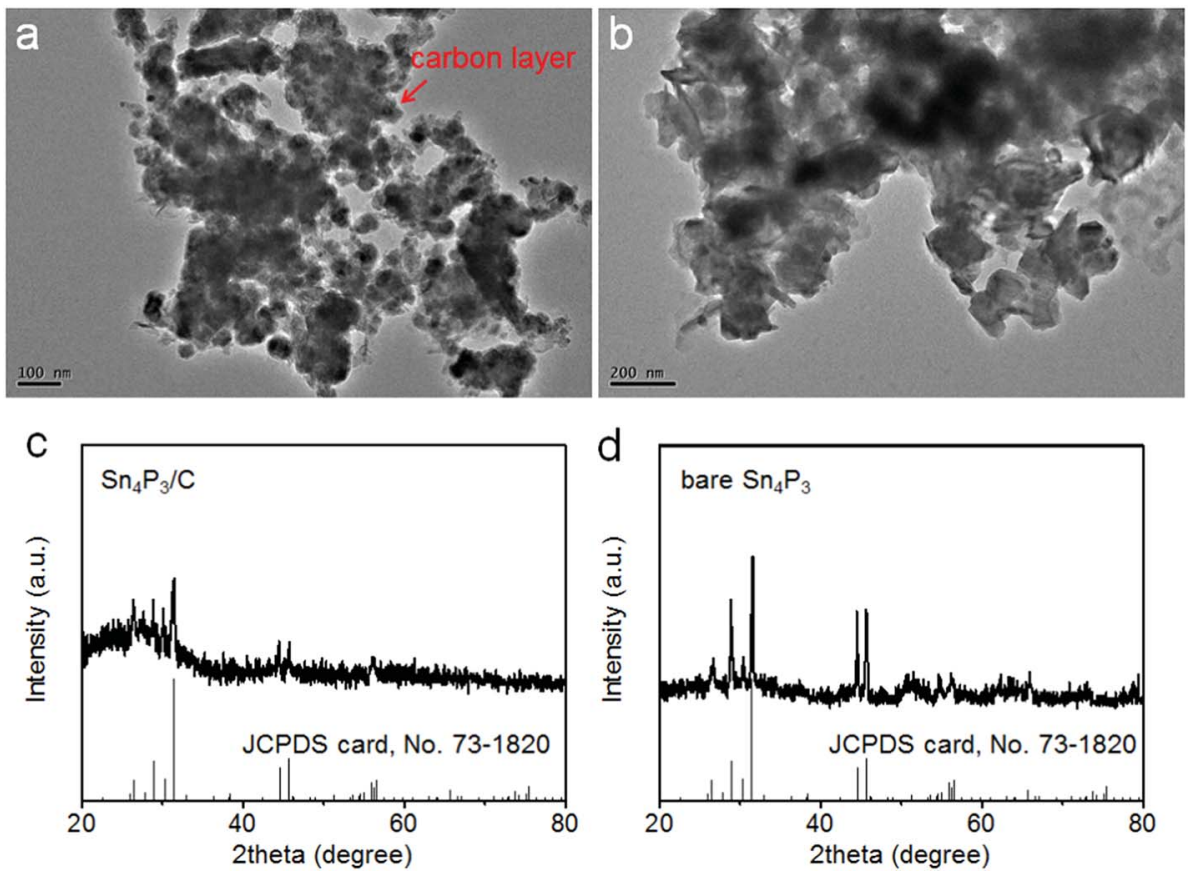

Fig. S2 Morphology and composition characterizations of $\mathrm{Sn}_{4} \mathrm{P}_{3} / \mathrm{C}$ and bare $\mathrm{Sn}_{4} \mathrm{P}_{3}$ as the control samples. (a) TEM image and (c) XRD pattern of $\mathrm{Sn}_{4} \mathrm{P}_{3} / \mathrm{C}$ material. (b) TEM image and (d) XRD pattern of bare $\mathrm{Sn}_{4} \mathrm{P}_{3}$.

The Royal Society of Chemistry apologises for these errors and any consequent inconvenience to authors and readers.

${ }^{a}$ Key Laboratory of Mesoscopic Chemistry of MOE, School of Chemistry and Chemical Engineering, Nanjing University, Nanjing, 210093, China. E-mail: mlb8976@126.com ${ }^{b}$ School of Chemistry and Chemical Engineering, Jiangsu University, Zhenjiang 212013, PR China. E-mail: shenyalong@njust.edu.cn ${ }^{c}$ College of Pharmacy, Inner Mongolia Medical University, Hohhot 010059, PR China

${ }^{d}$ MIIT Key Laboratory of Advanced Display Materials and Devices, Institute of Optoelectronics \& Nanomaterials, College of Materials Science and Engineering, Nanjing University of Science and Technology, Nanjing, Jiangsu, 210094, China 